\title{
Repensando las prácticas académicas: el diseño colaborativo de un proyecto de investigación a partir de la metodología IAP
}

\section{Rethinking Academic Practices: Collaborative Design of a Research Project Using PAR Methodologies}

\author{
Joan Roura-Expósito ${ }^{1}$ \\ Instituto de Ciencias del Patrimonio, CSIC \\ Antonio Luis Díaz Aguilar ${ }^{2}$ \\ Universidad Pablo de Olavide \\ Ana Ruiz-Blanch ${ }^{3}$ \\ Instituto de Ciencias del Patrimonio, CSIC \\ Cristina Sánchez-Carretero ${ }^{4}$ \\ Instituto de Ciencias del Patrimonio, CSIC \\ José Antonio Cortés-Vázquez 5
}

Universidade da Coruña/Investigador InTalent UDC-Inditex

\section{RESUMEN}

ParticiPat es un proyecto de investigación del Plan Nacional que tiene como objetivo analizar la participación y sus instrumentalizaciones en nueve estudios de caso sobre procesos de patrimonialización. A la hora de abordar el proyecto, quisimos cuestionar las jerarquías y lógicas raramente colaborativas que rodean la producción de conocimiento en la antropología. Para ello decidimos seguir los principios de la investigación acción participativa (IAP) para elaborar conjuntamente una guía de campo de mínimos que facilitara la comparabilidad de los casos de estudio. A través de la descripción de este proceso participativo reflexionamos sobre la estructuración de los proyectos de investigación, entendiendo que su arquitectura inicial condiciona la reproducción de lógicas de poder y autoridad entre las personas investigadoras. Asumimos así el reto de investigar sobre participación desde un meta diseño de investigación participativo que nos permite visibilizar algunos presupuestos ideológicos dentro de la práctica académica.

Palabras clave: Investigación y metodologías participativas; Autoridad; Horizontalidad; Privilegios epistémicos.

\footnotetext{
${ }^{1}$ Correo electrónico: joan.roura exposito@incipit.csic.es. ORCID iD: <https://orcid.org/0000-00022731-9113>.

${ }^{2}$ Correo electrónico: aldiaagu@upo.es. ORCID iD: <https://orcid.org/0000-0002-7111-3761>.

3 Correo electrónico: ana.ruiz blanch@incipit.csic.es. ORCID iD: <https://orcid.org/0000-00016632-9413>.

${ }^{4}$ Correo electrónico: cristina.sanchez carretero@incipit.csic.es. ORCID iD: <https://orcid.org/00000002-3900-3976>.

${ }^{5}$ Correo electrónico: jacorvaz@gmail.com. ORCID iD: <https://orcid.org/0000-0003-3566-7924>.
} 


\section{SUMMARY}

ParticiPat is a research project funded by the Spanish government which analyses participatory governance and its instrumentalisation with reference to nine different case studies of patrimonialisation. We sought to tackle the project in a way that would question the seldom collaborative hierarchies and rationales that tend to dominate knowledge production in anthropology. To this end, the team of researchers took the joint decision to apply the principles of Participatory Action Research (PAR) to collectively define minimum fieldwork guidelines that would allow us to compare the different case studies. In describing this participatory process, the paper reflects on the framing of research processes, showing how the initial structure conditions the reproduction of power relations and degrees of authority among researchers in the same team. By so doing, we have taken up the challenge of investigating participatory forms of governance using a metadesign that is also participatory, while rendering visible different ideological assumptions in academic practice.

Keywords: Participatory Research Methods; Horizontality; Authority; Epistemic Privilege.

\section{INTRODUCCIÓN}

En las últimas décadas, diversas corrientes de pensamiento han denunciado la vigencia de relaciones de poder y autoridad en el seno de la antropología. Estas perspectivas críticas han defendido la introducción de criterios éticos e intersubjetivos en la vinculación con los grupos investigados, desarrollando distintas propuestas teórico metodológicas como la antropología comprometida (Low y Merry 2010), antropología pública (Purcell 2000), etnografías militantes (Shukaitis y Graeber 2007), etnografía colaborativa (Lassiter 2005), investigación acción participativa (Greenwood y Levin 2000) o investigación co-operativa (Heron 1996). Como apunta Lamphere (2004), estas propuestas presentan numerosas similitudes ya que comparten ascendentes teóricos como Franz Boas, Ruth Benedict o Margareth Mead, tienen su origen geográfico en países del Norte global y se apropian de pensadores latinoamericanos que vinculaban investigación y transformación social desde los años 70 (Orlando Fals Borda, Paulo Freire, etc.).

Sin embargo, estas corrientes críticas han insistido básicamente en la importancia del compromiso, la implicación y la responsabilidad bacia los interlocutores, sin reconceptualizar las relaciones mantenidas dentro del ámbito académico, donde siguen reproduciéndose estructuras de desigualdad que relegan a la periferia de la disciplina opciones metodológicas alternativas o subalternas. De hecho, como ha señalado Bourdieu (2002), la producción académica está condicionada y determinada por disciplinas de pensamiento que consagran determinadas prácticas, saberes y metodologías. Solamente así se explica la paradoja de que algunos enfoques supuestamente participativos, cooperativos, simétricos, descentrados y horizontales se elaboren en contextos profesionales marcados por lógicas verticales, autoritarias, competitivas, centralistas e individualistas.

Partiendo de esta crítica, planteamos en este artículo una reflexión sobre las relaciones de poder naturalizadas en el diseño de los proyectos de investigación. Para ello, describiremos la elaboración colaborativa de la guía de campo del proyecto ParticiPat (acrónimo de Patrimonio y participación social: propuestas metodológicas y 
revisión crítica), elaborada por el conjunto del grupo de investigación vinculado al proyecto mediante el uso de herramientas de la investigación acción participativa (IAP). Al emplear principios de la IAP pretendíamos cumplir con tres objetivos teóricos y políticos. Primero, cuestionar las relaciones de autoridad dentro de los procesos de investigación, tanto aquellas que derivan de las posiciones académicas en el marco del proyecto (investigadora principal, investigadoras colaboradoras, becarias, personal de apoyo) como en referencia a un contexto de relaciones de poder más amplio (discriminaciones por género, edad, estatus profesional, modas académicas, etc.). Segundo, reflexionar sobre el reto de investigar y hablar de participación de forma participativa, incorporando a nuestro estudio el proceso de aprendizaje de estas dinámicas de trabajo. Tercero, poner en un primer plano elementos clave de la práctica investigadora que por lo general son invisibilizados, resaltando la centralidad de la afectividad en la co-construcción del conocimiento.

De este modo, pretendemos reflexionar sobre la brecha existente entre las intenciones que guían el desarrollo teórico en el campo de la participación y las prácticas concretas que se llevan a cabo dentro de los proyectos de investigación. No son pocas las expresiones populares que nos advierten de esta brecha: "predicar con el ejemplo", "aplicarse el cuento", "obras son amores", "del dicho al hecho hay mucho trecho", etc. Para quienes desarrollan estudios antropológicos, esta incoherencia supone uno de los rompecabezas principales a la hora de teorizar sobre las variables que entran en juego en las prácticas cotidianas de los grupos estudiados, así como el motivo de muchas cautelas metodológicas en el trabajo etnográfico. Este problema no solo atañe al objeto/sujeto de estudio y los procedimientos metodológicos aplicados en el campo, sino también al conjunto de procesos de debate, discusión y toma de decisiones que sucede en la trastienda de los proyectos de investigación. Por eso, consideramos importante poner el foco en las situaciones concretas de debate procedimental, un ámbito donde frecuentemente se generan coartadas, naturalizaciones e institucionalizaciones de determinados lugares de enunciación.

En consecuencia, en el proyecto ParticiPat nos planteamos el siguiente presupuesto de partida: la necesidad de un ejercicio explícito para generar desde el principio una metodología participativa y colaborativa. De hecho, si nuestro objetivo es analizar los procesos participativos de los otros en los diferentes estudios de caso, resulta oportuno que también analicemos nuestra propia praxis participativa en el seno del proyecto. En nuestro caso, hemos querido trabajar de manera colaborativa también por una cuestión experimental, puesto que nos ofrece la oportunidad de comprobar, en un contexto reducido y familiar, cómo los procesos participativos son herramientas de decisión que conllevan en sí mismas una serie de prácticas de gobierno (CortésVázquez, Jiménez y Sánchez-Carretero 2017).

Como parte de este proceso de elaboración colectiva de la metodología de trabajo, pusimos nuestras reflexiones en común en un archivo compartido, creando una especie de diario de campo grupal en una plataforma virtual que fue la savia y la semilla de este artículo. Las reflexiones volcadas en el espacio cibernético tras las distintas reuniones y procesos de toma de decisión mostraron elementos que normalmente la investigación no suele tener en cuenta o trata de soslayo, pero que aparecieron como fundamentales a la hora de pensar sobre qué investigar, y, sobre todo, para qué, cómo y en qué condiciones. En este artículo, usaremos las reflexiones del 
diario de campo grupal como testimonios, prescindiendo de la autoría individual de las voces entrecomilladas por decisión colectiva (votación mayoritaria no anónima) sin que esto implique una homogeneidad en las posiciones de las participantes. Usaremos la primera persona del plural para remarcar la centralidad del consenso en el habitus político del proyecto ParticiPat. Entre los acuerdos tomados por consenso figuran la propia participación en la escritura del artículo, la decisión sobre su autoría y el uso de substantivos genéricos, como personas, en la redacción final. Esta última decisión se tomó después de un debate grupal, primero presencial y después virtual, sobre el tratamiento del género en la escritura. El acuerdo fue intentar utilizar formas de lenguaje que soslayaran la atribución genérica y apostar por el uso del femenino cuando la elipsis fuera complicada a nivel sintáctico.

\section{EL PROYECTO PARTICIPAT Y LA LÓGICA DE LA PARTICIPACIÓN EN INVESTI- GACIÓN}

Para poder contextualizar nuestro análisis, incluimos primeramente un resumen del proyecto ParticiPat ${ }^{6}$. El proyecto se centra en las instrumentalizaciones de diferentes procesos participativos en gestión patrimonial, planteándose un triple objetivo: (1) analizar el papel que están jugando las técnicas participativas en políticas patrimoniales; (2) analizar el vínculo entre participación pública y procesos de patrimonialización; (3) analizar el vínculo entre dicha participación e instituciones patrimoniales. El proyecto reúne un total de 16 personas investigadoras procedentes de 6 instituciones diferentes que desarrollan un total de 9 estudios de caso (Sánchez-Carretero y Jiménez 2016: 195-196).

A nivel teórico metodológico, el proyecto ParticiPat asumió desde un primer momento la crítica a las formas de producción de la antropología dominante (Restrepo y Escobar 2005), cuestionando la organización contemporánea del campo intelectual, el mito del pensamiento autónomo y los privilegios epistémicos derivados de la posición teórica o académica. La apuesta por la metodología IAP en la elaboración de la guía de campo pretendió precisamente democratizar y horizontalizar el proceso de construcción de saberes. Más allá de contraponer nuestra experiencia a una supuesta antropología hegemónica impregnada por una razón moderna, colonial, capitalista, patriarcal, occidentalocéntrica y cristianocéntrica (Grosfoguel 2013), nuestra aportación pretende diversificar y enriquecer el espacio epistemológico político de la disciplina. Varias reflexiones compartidas en el diario grupal apuntan a la estrechez de los regímenes de conocimiento antropológico, problematizando especialmente la prevalencia de relaciones de poder, jerarquía y autoridad en el mundo académico:

La forma participativa ha sido una apuesta consciente por la colaboración en un contexto académico muchas veces regido por el individualismo y la competencia. "El diseño de la guía ha retado al academicismo empeñado en mantener jerarquías entre actores". "Sentí que la participación rompía con los esquemas jerárquicos de categoría, experiencia profesional y grado de conocimiento de los miembros". "El diseño participativo ha sido un

\footnotetext{
${ }^{6}$ Para conocer en detalle los estudios de caso, objetivos e investigadoras de ParticiPat se puede consultar la página del proyecto: <http://participat.org/>.
} 
instrumento útil para superar la ideología del liderazgo, así como todos los sesgos, marginalizaciones y desequilibrios relacionados con el pensamiento teórico y el peso académico del IP (investigador principal)". (Diario de campo grupal, 1-15 mar. 2016).

Por esta razón, desde el comienzo del proyecto se pusieron sobre la mesa distintas precarizaciones de las integrantes del mismo. En este caso, como consecuencia de las condiciones laborales débiles e inestables de algunos miembros de ParticiPat, a quienes no se reconoce como investigadoras del proyecto, así como por su agravamiento derivado del recorte en un $75 \%$ del presupuesto concedido por el Ministerio. De hecho, de las 16 personas participantes, en un principio solo dos fueron reconocidas como miembros del equipo de investigación por no cumplir el resto los requisitos de contratación que exige el Ministerio; el resto formaban parte del equipo de trabajo. Estas precarizaciones, además de afectar a las condiciones materiales y simbólicas de las investigadoras para poder desarrollar sus vidas (Pérez Orozco 2014), forzaron una readaptación de las estrategias del grupo, una minimización de los objetivos, los casos de estudio inicialmente propuestos, los recursos y los tiempos para hacer trabajo de campo y, por supuesto, las expectativas de impacto social del proyecto.

\section{LOS PRESUPUESTOS IDEOLÓGICOS DE LA IAP}

Las relaciones de la IAP con la antropología/etnografía son muchas y multidimensionales. El relativismo y holismo propios de la tradición etnográfica fue conformando el magma del que emerge la riada de posicionamientos que subvirtieron la relación sujeto/objeto a partir de los años 80 en antropología ${ }^{7}$ y que conecta con los principales postulados de la IAP. En estos años precisamente se consolida la "antropología comprometida y la antropología colaborativa" (Schensul y Schensul, en Low y Merry 2010; Lassiter 2005), corrientes comprometidas con la dignidad de todos los seres humanos y la justicia social (Low y Merry 2010: 204) - y, por tanto, la transformación social-, las cuales defienden las relaciones de colaboración e igualdad en el trabajo de campo, es decir, la gestión compartida de un proyecto de investigación. En este punto, la IAP sería un ejemplo de esta forma de colaboración (Low y Merry 2010: 209), lo cual nos introduce en un interesante debate sobre las áreas de encuentro o las diferencias existentes entre ambos enfoques (Berraquero Díaz, Maya Rodríguez y Escalera 2016). En el caso concreto de este artículo, a la hora de diseñar el proyecto de investigación ParticiPat no estábamos haciendo etnografía comprometida, ya que el trabajo de campo etnográfico no había empezado, sino que quisimos adoptar los presupuestos de la IAP para diseñar conjuntamente la estructura del proyecto.

Tomada esta decisión profundizamos en esta propuesta metodológica y vimos que no hay consenso sobre sus precedentes concretos. Algunos señalan a Kurt Lewin y sus trabajos de psicología social que versan sobre la involucración del investigador en los problemas sociales. Otros a John Dewey, ligado al campo de la pedagogía y

\footnotetext{
${ }^{7}$ El paralelismo con lo ocurrido en otras ciencias sociales es, nunca mejor dicho, paradigmático. Véase, por ejemplo, Ibáñez (1985), Fals Borda (1985) o Rodrigues Brandão (1986).
} 
su defensa de la figura del maestro como investigador. Hay otros que identifican los orígenes de la IAP con John Collier, comisario del Indian affairs en Estados Unidos, por enfrentar los problemas de relaciones interétnicas entre indios, administradores, letrados e investigadores. Otros miran más atrás y consideran, por ejemplo, a Alexander Bain, del movimiento de la Ciencia en Educación, o a Karl Marx con su Encuesta Obrera, como instrumento de reflexión sobre las condiciones de vida del proletariado, como algunas de las finas raíces que entroncan con el corpus teórico práctico de la IAP que comienza a desarrollarse a partir de los años 70 del siglo XX (Cortesão 2006: 183-189).

En América del Sur, podemos tomar como primera referencia y cuasi fundacional el trabajo de Paulo Freire (1997 [1970]). Allí también se desarrollaron investigaciones por parte de autores como Orlando Fals Borda, Carlos Rodrigues Brandão o Paulo Latapi, que fueron estructurando un grupo latinoamericano de IAP, en principio muy vinculado a la educación popular pero pronto abiertos a otras problemáticas de actuación, cuyos contrastes teóricos y metodológicos tuvieron una primera formulación académica colectiva importante con el I Simposio Mundial sobre Investigación Acción y Análisis Científico, realizado en Cartagena de Indias en 1977 (Fals Borda 1978; Vejarano 1983; Cortesão 2006). Por otra parte, en Europa, más que en Estados Unidos - la Guerra Fría y las inversiones en un tipo de investigación y no en otro son un buen referente de la tesis de Habermas sobre la ciencia como ideología一, se fueron conformando grupos de investigación acción participativa influenciados por los enfoques de Lewin, sobre todo en la denominada Democracia Industrial Noruega y en el Instituto Tavistock de Relaciones Humanas de Londres.

Debido a estas diferencias entre los entornos industriales europeos de los años 70 y 80 y las "circunstancias dramáticas" de América Latina (Fals Borda 1978), junto al propio desarrollo ideológico práctico de la IAP en unos contextos y otros, para esta primera etapa de desarrollo de la IAP se suelen distinguir dos tradiciones principales, dos extremos entre los que se pueden situar todas las escuelas que toman como punto de referencia este marco de análisis e intervención. De un lado, está la "pragmática operativa", en cuyo caso la participación es sobre todo un método para la resolución de problemas grupales o institucionales concretos, puntuales, sin un análisis de sus relaciones con el conjunto del sistema social y con etapas fijas sin recorridos posteriores y, por tanto, sin alternativas globales. De otro lado, está la tradición "crítica implicativa", que propugna la investigación acción a partir de un rechazo global de las situaciones de desigualdad, discriminación, exclusión, pobreza, etc. existentes en cualquier sociedad y, por tanto, como una vía de liberación social (Colectivo Ioé 2003: 6-11).

Esta circunstancia ha generado cierta asociación connotativa entre una IAP del Norte (anglosajona), con una orientación más pragmática, y una IAP del Sur (latinoamericana), con un sentido más revolucionario. División no exenta de críticas por su simpleza y ubicación en primeros y terceros mundos (Escalera y Coca 2013: 26) y por dejar atrás interesantes experiencias en África, Filipinas, India, Italia, España o Francia. Valgan como ejemplos las diversas escuelas de intervención social directa o indirectamente relacionadas con la IAP surgidas tras el Mayo del 68 en Francia: "el análisis institucional" de Lourau, Lapassade o Guattari, "la crítica institucional y creatividad colectiva" de Michel Seguier, el "sociopsicoanálisis" de Gérard Mendel o la "sociología permanente" 
de Alain Touraine. O también el núcleo constituido en Madrid en torno al «socioanálisis" de corte dialéctico y hermenéutico de Jesús Ibáñez, por no mencionar el desarrollo de algunas Universidades Populares y la Educación de Adultos en España en los años 80 (Colectivo Ioé 2003: 8-14; Red CIMAS 2015: 16). Y previamente, lo cual nos sitúa en los antecedentes mencionados anteriormente, el modelo educativo de las escuelas populares republicanas españolas y su conexión posterior con movimientos contestatarios en América Latina.

En esta contextualización histórica pueden vislumbrarse cuáles y de qué tipo son los principios estructurantes de la IAP. En primer lugar, se parte de la necesidad de cambiar el rol del investigador, como observador distante, por un investigador involucrado en la resolución de problemas concretos, principio sostenido por Lewin (Greenwood y Levin 2007: 17). Sin embargo, es importante no olvidarse del para qué y para quién sirve esta episteme (Red CIMAS 2015: 17). A diferencia de la ciencia convencional y su pretensión de independencia, la IAP reivindica su carácter comprometido y su vocación transformadora, superando meros posicionamientos éticos para situarse en la lucha contra las desigualdades y las estructuras que sustentan y reproducen la dominación (Escalera y Coca 2013: 25-26). Este sesgo político e ideológico constituye otro de sus principios epistemológicos.

Más aún, contrariamente a la investigación social aplicada clásica llevada a cabo por los expertos, la relación con la práctica y con los otros que propugna la IAP no se desarrolla de forma vertical y experta, sino de manera intersubjetiva, superando la relación objeto/sujeto. Esta premisa nos obliga al diálogo de saberes, otro de los principios de la IAP, y a un intento de construcción de una entidad colectiva de investigación acción que protagonice los procesos de producción de conocimiento y el cambio para la autotransformación desde la praxis. Por tanto, la conexión entre teoría y práctica, conocimiento y aplicación, pensamiento y acción ha de ser una guía constante en los procesos de IAP (Colectivo Ioé 2003: 6-7; Escalera y Coca 2013: 24-25).

Tal y como explican Escalera y Coca (2013: 26-27), la IAP no es una teoría, puesto que bebe de corrientes teóricas y filosóficas distintas (marxismo, anarquismo, constructivismo, hermenéutica). Tampoco es una disciplina, puesto que su vocación es la transdisciplinariedad. Ni tan siquiera viene definida fundamentalmente por una metodología, puesto que se trata más bien de un armazón epistemológico para orientar procesos colectivos de investigación e intervención. Su carácter único viene definido por un objetivo principal: propiciar y desarrollar procesos participativos, articulados por las co-decisiones y las co-responsabilidades (Escalera y Coca 2013: 17-38). La elección de metodologías y técnicas determinadas busca ser coherente con dichos principios, puesto que las técnicas en sí mismas no ofrecen garantías de que los procesos sean participativos. De hecho, en no pocas ocasiones el recetario técnico de la participación ha terminado por cumplir una función cosmética (Coca 2008; Quintero 2011; Sánchez-Carretero 2013), por camuflar mecanismos de cooptación ejercidos desde el mundo empresarial, como tempranamente se observó en las grandes empresas escandinavas (Greenwood y Levin 2007: 17), o por ser la excusa y justificación para imponer decisiones tomadas previamente desde la maquinaria del Estado, como ha ocurrido, por poner un ejemplo del otro extremo de Europa, con los Planes de Desarrollo Sostenible en los Parques Naturales Andaluces, donde los procesos participativos se convirtieron en escenarios en los que los actores sociales, después de cons- 
truir una obra colectiva, eran despreciados por los políticos situados detrás del telón (Coca y Díaz 2005; Coca 2008). Como reacción a esta situación, se propuso una "Cosmovisión participativa" (Reason 1994), es decir, una intención previa desde la cual implementar una metodología participativa.

Este posicionamiento, desarrollado por el grupo de Human Inquiry (Human Inquiry, Collaborative Inquiry, Action Inquiry y Cooperative Inquiry) (Greenwood y Levin 2007: 208-212), nos introduce explícitamente en el análisis de las dimensiones éticas, emocionales y motivacionales contenidas en los procesos de investigación y, por tanto, existentes en las relaciones personales que se establecen en ellos. Este análisis es central para la reflexión que planteamos en este artículo sobre la generación participativa de conocimiento, tanto desde un punto de vista práctico como ideológico. Partimos de la idea de que el conocimiento es construido a partir de secuencias y combinaciones de diferentes tipos de saber y acciones en un contexto determinado. Reason (1994) nos habla del conocimiento vivencial, presentacional, proposicional y, finalmente, del práctico. Estas diferencias resultan particularmente fructíferas, ya que atendemos a las actividades diversas que forman parte de un concepto amplio e inclusivo del conocimiento y nos sitúa en una perspectiva procesual, reflexiva e introspectiva.

La construcción del conocimiento es una acción colectiva que deriva de la interpretación que las personas hacen de sus vidas cotidianas, en interacción con el resto de actores sociales y con la realidad contextual que viven en un momento y lugar dados (Lucio Villegas 2006: 151). La transformación social requiere la transformación personal, hasta el punto de que, como defiende Torbert (2001: 250-260), las reflexiones surgidas en el desarrollo de las discusiones y su análisis posterior, permiten problematizar los patrones interpretativos desde los que actuar. Para Marshall (2004) tanto la atención al otro (attentional disciplines), como la mejora del autoconocimiento, es prerrequisito y constituyen una buena motivación para optar por la IAP.

\section{MICROPOLÍTICA DE UNA REUNIÓN DE PROYECTO}

En esta sección describiremos cómo aplicamos las premisas de la IAP al proceso de elaboración de la guía de campo común del proyecto. En primer lugar, describiremos las distintas sesiones de trabajo, para posteriormente reflexionar sobre las aportaciones de la metodología IAP, así como algunas de sus condiciones de posibilidad y limitaciones en la práctica académica. De este modo, atendemos a la dimensión micropolítica $^{8}$ de la práctica investigadora, entendida como la concreción efectiva de la gobernanza en su dimensión molecular, es decir, los aspectos internos, cotidianos e informales de la actividad investigadora durante la fase de diseño del proyecto.

\section{PERSONAS, CIRCUNSTANCIAS Y HECHOS}

En febrero de 2016 tuvo lugar la primera reunión del proyecto ParticiPat, en la

\footnotetext{
${ }^{8}$ Para una aproximación al desarrollo filosófico del término micropolítica, véase Guattari y Rolnik (2006); y para conocer su aplicación teórica y metodológica en el campo educativo, véase Ball (1989).
} 
sede del Incipit, en Santiago de Compostela. Estaban convocadas tanto las personas que llevaban a cabo los estudios de caso como las que trabajaban en otras tareas dentro del proyecto, incluyendo quienes colaboraban haciendo estudios de tipo teórico y quienes hacían funciones de apoyo a la investigación y administrativas. Las edades, situaciones laborales y posiciones dentro del ámbito académico también eran muy diversas. Por ejemplo, algunas investigadoras consolidadas superaban en más de treinta años a otras investigadoras predoctorales y personal de apoyo, mientras otras en formación predoctoral superaban en edad a personas con carreras consolidadas. Las condiciones de precariedad laboral de la mayoría de las personas investigadoras que colaboraban en el proyecto impedían su vinculación oficial como parte del grupo de investigación, relegándolas — de acuerdo a la normativa vigente- a la figura de grupo de trabajo. De hecho, solo dos de las investigadoras consolidadas, contando a la IP, pudieron formar parte del grupo de investigación de manera oficial. El resto de las 16 personas no tenían ese estatus, en su mayor parte por carecer de un contrato vigente durante la totalidad de la duración del proyecto; un requisito de los proyectos de I+D del Plan Nacional financiados por el Ministerio de Ciencia, Innovación y Universidades, que es el organismo del que depende la investigación en España.

Esta era la primera vez que nos reuníamos para trabajar en grupo. A pesar de que muchas de nosotras nos conocíamos de otras colaboraciones, eventos y proyectos, otras se veían por primera vez. De las 16 personas que formaban parte del proyecto, acudieron a la reunión 14 . De ellas, 5 tenían una situación consolidada en instituciones académicas (Universidad de Santiago, Universidad Pablo de Olavide, IAPH y CSIC), otras 5 tenían salarios precarios vinculados a instituciones académicas y 4 vivían en situación precaria sin contratos laborales. Entre otras cosas, el desequilibrio entre investigadoras que podían ser reconocidas oficialmente por el Ministerio frente a las que no ( 2 frente a 16) hizo que el tema de los desequilibrios en la investigación atravesara la propia dinámica grupal.

El objetivo de esta reunión era contextualizar y facilitar la elaboración de una guía de mínimos para el trabajo de campo con referencias teóricas y etnográficas compartidas, así como anotaciones relativas a la diversidad de situaciones a analizar. El trabajo colaborativo que se analiza aquí es, por tanto, previo al desarrollo de las diferentes etnografías de los estudios de caso. La finalidad última era establecer unas bases comunes para que los estudios de caso del proyecto pudieran ser sometidos a las mismas preguntas de investigación. Para ello se organizaron varias sesiones con propósitos específicos.

En la primera sesión matutina, un grupo de investigadoras hizo una presentación del trabajo previo realizado en torno a las instrumentalizaciones de la participación en distintos ámbitos de estudio. El objetivo era introducir elementos de análisis y reflexión sobre las posibilidades que ofrecen los procesos participativos, mediante esquemas y cuadros de ideas. Este trabajo se enmarcaba dentro del Laboratorio de Ideas desarrollado durante los primeros meses del proyecto (septiembre a diciembre de 2015) y en el que colaboró tanto el personal del Incipit vinculado a ParticiPat, como una compañera del Estudio Rurban. Se escogió la etiqueta Laboratorios de Ideas para describir la forma de construcción teórica, grupal, del proyecto. En estos laboratorios se hizo una revisión de la bibliografía y webografía sobre estudios similares centrados 
en los usos de procesos participativos vinculados a la gestión patrimonial, y también sobre otras áreas como políticas públicas, medio ambiente, cooperación, etc. Después de esta puesta en común inicial, se hicieron las presentaciones de los diferentes estudios de caso por parte del resto de integrantes del grupo.

Para diseñar la guía de campo de mínimos, se partió de la lectura previa de «El taller del etnógrafo" (Díaz de Rada 2011), con el objetivo de entender las formas de co-construcción de las categorías analíticas. Sin embargo, a diferencia del ejemplo presentado en este libro, donde una propuesta individual era discutida, consensuada y asumida por el resto del grupo, nuestra idea era que la propuesta inicial fuera generada a través de la dinámica grupal. Para conseguirlo, se decidió seguir los fundamentos de la IAP a la hora de estructurar la participación en la actividad de construcción de la guía. Los límites y posibilidades de esta forma de trabajar se pusieron enseguida de manifiesto, surgiendo las primeras reflexiones en torno a las coherencias o incongruencias entre el fondo y la forma, que a su vez nos llevaron a plantear cuestiones de jerarquías y horizontalidades, no solo en entornos participativos, sino en la propia práctica académica: ¿de qué forma la manera de producir conocimiento condiciona los resultados?, ¿cómo repensar la autoridad académica en el diseño de la investigación?

Finalizado este primer bloque de trabajo, y tras una comida colectiva, se inició por la tarde el taller participativo para la elaboración de la guía. La técnica utilizada fue la visualización de ideas con tarjetas o cartulinas de tamaño medio, de un modo parecido a como se utilizan en trabajos de IAP (ver Figura 1). En las tarjetas, cada participante podía expresar sus opiniones por escrito sobre distintas cuestiones relacionadas con los objetivos propuestos para este taller. Estas opiniones eran recogidas por dos moderadoras que las situaban en un soporte visible y accesible, encargándose también de organizar, clasificar y matizar (en caso de dudas), las opiniones según los contenidos. Las dos personas del equipo que ejercieron de moderadoras y dinamizadoras comenzaron explicando brevemente el uso y el sentido de esta técnica, conectándola con el objetivo fundamental del encuentro: llegar a un acuerdo de mínimos en la guía de campo. Para ello se trabajó sobre tres grandes temas:

- Selección de tres ejes analíticos sobre procesos participativos en contextos patrimoniales.

- Selección de tres elementos fundamentales de cada eje analítico.

- Selección de conceptos clave para guiar las orientaciones analíticas.

A continuación, las dinamizadoras entregaron tres tarjetas a cada persona, a las que se les preguntó por los ejes analíticos que se podrían establecer, incidiendo en la importancia de la concisión y la síntesis en las respuestas. Recogidas y barajadas dichas tarjetas anónimas se comenzaron a leer, colocar y agrupar en la pared. Tras algunas deliberaciones sobre el conjunto de tarjetas, debido a la existencia de tarjetas más teóricas y otras más metodológicas, se estableció finalmente el siguiente cuadro analítico:

- Mapa/red de actores

- Aportes y retornos (inputs, outputs y outcomes)

- Procesos y participación (formas, tipos, cauces)

- Contexto (diagnóstico de las circunstancias socioeconómicas y culturales del objeto o motivo de la participación)

- Versiones del patrimonio (discursos, significados, interpretaciones, usos, narrativas) 


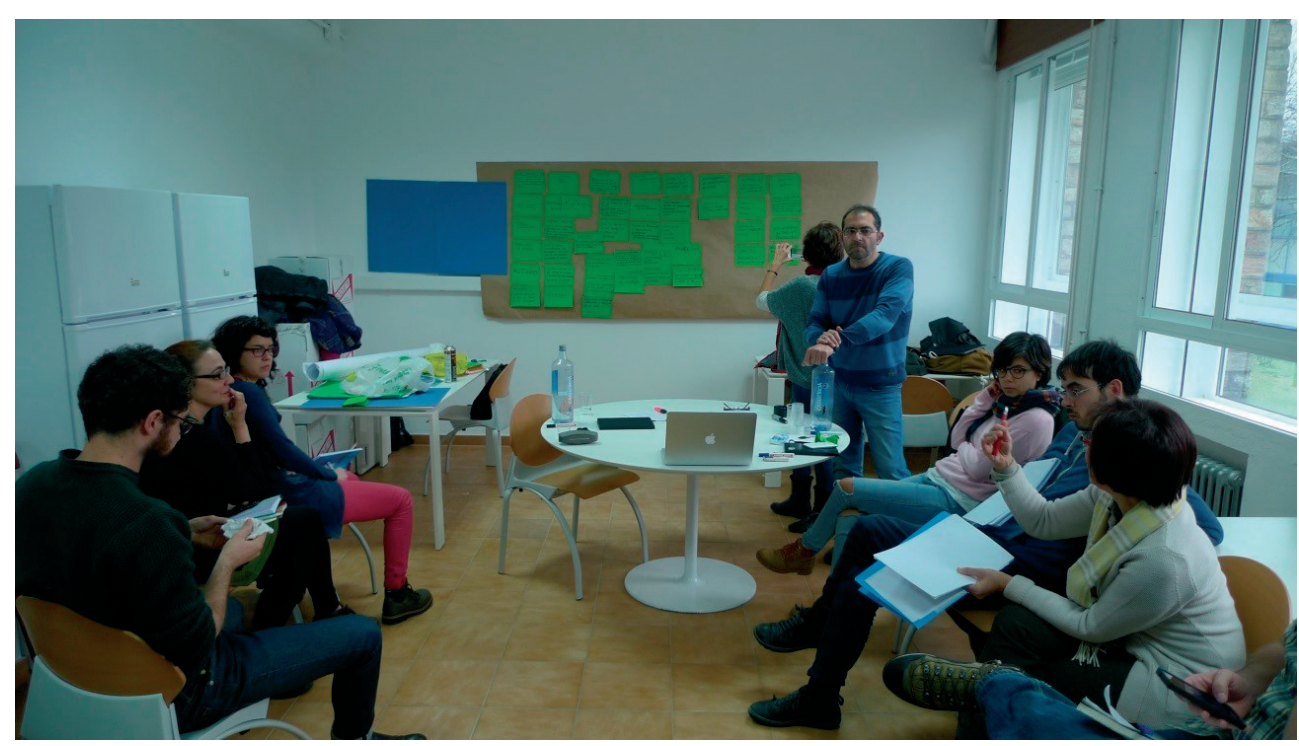

FiguRA 1.-Sala de reuniones. Autoría: María Masaguer

En la segunda sesión de trabajo se entregaron otras tres tarjetas a cada participante y se les pidió que, una vez escritas las ideas, las explicaran en unos cinco minutos. Lógicamente se perdió el anonimato y ganó fuerza la expresión oral de los participantes. En cualquier caso, la confianza generada durante la dinámica grupal del día anterior fue clave para fortalecer la horizontalidad y la participación.

Cuando estaba finalizando la ronda de entrega de estas tarjetas, una compañera propuso un esquema de análisis más sintético y comprensivo de presentación de resultados. Dibujando los trazos en una pizarra de la sala estableció un eje analítico a partir de líneas de tiempo paralelas en torno a los procesos de patrimonialización, participación e investigación, con hitos, fases y dimensiones espaciales y cartográficas. Con esta base construimos un segundo esquema síntesis de lo que había ido mostrándose en las tarjetas, que fue debatido y puesto en común. Finalmente, cerramos la reunión con la cuarta sesión, ideada para programar las siguientes tareas del proyecto?.

Las modificaciones de aplicación de la técnica que realizamos durante la reunión nos llevaron a analizar cómo la IAP no puede ser únicamente un instrumento de aplicación mecánica y reproducible de forma idéntica en circunstancias cambiantes, sino un proceso siempre abierto de negociación y conflicto, donde la práctica cobra más relevancia que la teoría y lo real prevalece sobre lo ideal. La técnica utilizada durante la reunión de Santiago buscaba facilitar la participación de un grupo que se estaba conociendo. El hecho de ser por escrito facilitaba el anonimato, dificultaba el

\footnotetext{
${ }^{9}$ El informe completo de la reunión con la guía de mínimos está disponible en el repositorio del CSIC (<http://digital.csic.es/handle/10261/144448>).
} 
monopolio de la palabra y garantizaba que todas las voces estuvieran presentes. Al visualizarse las ideas de todas las participantes se obtenía una panorámica general de las diversas opiniones de una manera transparente. Esto permitía agrupar las ideas por bloques y realizar una gradación de las problemáticas, así como construir acuerdos e identificar desacuerdos.

El objetivo final era fomentar la reflexión colectiva desde la consciencia de que una técnica en sí misma no garantiza un proceso participativo, ya que este debe ser diseñado atendiendo a sus fundamentos ideológicos. No se trataba de aplicar una receta, sino de encuadrar el estudio en el marco general de la IAP, donde eran fundamentales valores e intenciones como la consideración de todas las personas como sujetos activos y con capacidad de auto-organización, la integración de los sectores minoritarios, la transparencia en el diseño y la corresponsabilidad en la toma de decisiones.

En nuestro caso concreto, desatendimos algunas máximas de este tipo de talleres porque el foro era distinto al que solemos encontrar cuando diseñamos una IAP. En primer lugar, nosotras teníamos un conocimiento previo de la metodología y habíamos realizado una serie de tareas preparatorias de la reunión. En segundo lugar, las personas encargadas de la dinamización también escribieron sus propias tarjetas, lo que altera su papel de facilitadoras del proceso participativo y la máxima de no generar opiniones propias sobre quienes participan. En tercer lugar, la reunión fue entre compañeras del grupo de investigación que, por otra parte, comparten un bagaje disciplinario común. Además, el desarrollo de las sesiones no se ajustó al tiempo previsto y, por tanto, no se llevaron a cabo todas las prácticas inicialmente planeadas. Sin embargo, esta flexibilidad metodológica estuvo al servicio de los fundamentos ideológicos de la IAP, y, a nuestro juicio, facilitó la comunicación y el involucramiento de todas las investigadoras.

Protagonizar este proceso nos ha situado en una perspectiva etnográfica propia, desarrollando lo que podríamos denominar una subjetividad participativa. A nivel teórico nos sentíamos cómodas con enfoques fenomenológicos que valoran la experiencia corporal y empírica como requisito previo a la teorización y el ejercicio intelectual. Practicar la participación en gerundio, nos ha permitido comprender, desde nuestra propia vivencialidad, las interdependencias entre fondo y forma, la articulación entre teoría y práctica, y, en definitiva, las implicaciones sensitivas, corporales y afectivas de la metodología IAP.

\section{RELACIONES, AUTORIDADES Y AFECTOS}

La dinámica de trabajo estuvo orientada hacia la búsqueda de la horizontalidad, tanto en la toma de decisiones como en la forma de utilizar los espacios. El hecho de sentarnos en semicírculo en la sala o intercambiar las posiciones tras cada descanso rompió con el embalaje académico habitual. A estas ganas de experimentar con el espacio, el tiempo y los vínculos establecidos en el proyecto, subyace la intención de diversificar y enriquecer el espacio epistemológico político de la disciplina, abriéndolo a la cooperación.

Nos parece necesario subrayar que el proceso colaborativo desarrollado durante 
la elaboración de esta guía de campo no implica para nada la eliminación de determinadas autoridades, sino la multiplicación de las mismas. En nuestro proceder buscábamos transformar la estructura jerarquizada impuesta por la entidad financiadora que, con su diferenciación de distintas figuras (IP como la principal de ellas) y el establecimiento de condiciones para la participación en el grupo de investigación, parece seguir el clásico esquema weberiano, otorgando poder a un solo individuo (Castro y Rodríguez 2009: 108).

Con esta propuesta, el liderazgo que se otorga a una sola figura o grupo se extiende al total de las personas que participan en el proyecto de investigación. Así, más que considerar como referencia la autoridad tradicional legitimada por la costumbre y las prácticas aceptadas en investigación, o la autoridad burocrática legal, basada en el cumplimiento de las regulaciones impuestas por la entidad financiadora, concebimos la autoridad grupal como una autoridad carismática legitimada por los atributos personales de cada individuo, a los que el resto considera capaces de interpretar su voluntad y realizar lo que conviene para el bien común (Weber 1978), obteniendo como resultado una tenencia y delegación del poder (la capacidad de participación en este caso) por parte de cada persona participante en el proyecto de investigación.

Con la metodología propuesta, pretendíamos también repensar las formas de funcionamiento dominantes en la academia, donde se normaliza que una voz inicie y dirija el proceso, a modo de autoridad tradicional o burocrática legal. Esta propuesta alternativa de participación horizontal promovía en cada una de las personas del proyecto de investigación el entendimiento y apropiación de las subjetividades de sus compañeras, desde la colaboración y no la competencia. Como comentaba una de las investigadoras:

Poder llegar a un consenso de mínimos para categorizar y parcelar la realidad que queremos investigar supone un sano ejercicio de renuncia o matización del propio ego para abrazar e in-corporar los otros egos que componen el grupo [...] El aprendizaje conjunto y consensuado con las demás subjetividades, sin tratar de imponer nuestras versiones y visiones de la realidad, es un ejercicio muy beneficioso para la musculatura etnográfica (Diario de campo grupal, 1-15 mar. 2016).

A las reflexiones sobre los niveles de precarización y las distintas relaciones de poder dentro del grupo, queremos sumar también un análisis de los afectos implicados en el proyecto de investigación. Por un lado, hicimos un esfuerzo por visibilizar nuestras propias inseguridades y vacilaciones como investigadoras, valorizando las capacidades generativas de la duda y reclamando cierto derecho a la incertidumbre en un campo de enorme flexibilidad hermenéutica como la participación. Por otro lado, compartimos nuestras ideas, dudas, miedos, sugerencias, preguntas e incertidumbres, para evitar el oscurecimiento del pensamiento bajo criterios de autoridad académica. En este sentido, el juego y el ambiente relajado se mencionaron en el diario grupal como ingredientes esenciales de una praxis colaborativa:

Las dudas, los miedos, las incertidumbres se superan mejor desde opciones colaborativas, compartiendo ganamos todos, también en ánimos y seguridad para echar a andar. La ansiedad derivada de la toma de decisiones individual se alivia enormemente cuando el proceso de decisión es compartido (Diario de campo grupal, 1-15 mar. 2016). 
La dinámica de trabajo estuvo también orientada hacia la búsqueda de la horizontalidad en otros momentos que no son técnicamente participativos, en esos otros tiempos no académicos que, sin embargo, están siempre presentes en este tipo de reuniones académicas: comidas, descansos, trayectos, etc. Estos espacios no productivos pueden ser pensados en términos goffmanianos como el bastidor de la escena, lugares no visibles desde el escenario donde se ensaya y planifica la representación, siendo fundamentales para facilitar el conocimiento mutuo y fortalecer la dinámica grupal. Por su carácter intersticial, entre lo público y lo privado, las relaciones establecidas en el tiempo de ocio posibilitaron una mayor vinculación afectiva entre los miembros del grupo, que sin duda repercutió positivamente en el proyecto. En última instancia, el reconocimiento de nuestras inseguridades, dilemas y precariedades en espacios y tiempos informales cobró tanta relevancia como para ser tenidas en cuenta en espacios formales de toma de decisiones. Estas reflexiones entroncan con otras dimensiones humanas que raramente se incorporan en el análisis, como el papel de la confianza, la seguridad o la afectividad en el trabajo colaborativo.

\section{CONCLUSIONES}

Hace tiempo que la antropología defiende que las aportaciones de una investigación no dependen únicamente de su resultado final, sino también de la forma de alcanzarlo, convirtiendo el dilema filosófico sobre si los fines justifican los medios en un absurdo, en la medida que el método es un objetivo en sí mismo. En un momento en el que tanto se habla de sensibilizar la etnografía y del lugar de la experiencia en la construcción de habitus y subjetividades, parecía imprescindible que un proyecto sobre participación adoptara a su vez un diseño participativo, entendiendo la participación como una herramienta metodológica, un instrumento de investigación, una técnica proyectiva y un vector de conocimiento.

La elaboración colaborativa de la guía de campo del proyecto ParticiPat tenía precisamente como objetivo replantear una dinámica que, por tradiciones académicas e imposiciones reglamentarias, tiende a reproducir jerarquías dentro del trabajo investigador. El resultado de este proceso fue una forma diferente de llevar a cabo el diseño de una investigación, asumiendo debates introducidos por los propios estudios de participación y, simultáneamente, resaltando elementos clave de la práctica investigadora como la afectividad, la inseguridad o la precariedad profesional que muchas veces resultan invisibilizados.

El trabajo aquí analizado nos conecta así con el concepto central de Paulo Freire de "seres en situación" (1997 [1970]: 135), de nuestra comprensión y acción sobre la base de nuestra percepción in situ de las circunstancias que vivimos, y con la propuesta de Gramsci del necesario cambio intelectual y valorativo para edificar una nueva ideología. En la socio praxis se parte de la desigualdad existente para construir procesos transformadores (Red CIMAS 2015: 18). Dadas las diferencias de poder, estas tienen que ser reconocidas para permitir el comienzo del trabajo colectivo, pero combatidas en el curso del mismo. En la IAP los grupos han de trabajar conjuntamente para cambiar su lenguaje, sus modos de acción, sus relaciones sociales y, así, prefigurar, presagiar, provocar cambios en el marco más amplio de las interacciones que caracterizan nuestra sociedad (Macttagart, en De Souza 2007: 129 130). En este artículo, hemos planteado 
la necesidad práctica de aplicarlo también al trabajo que desarrollamos como profesionales de la investigación desde la universidad y otras instituciones académicas.

El uso de la metodología IAP en la generación de las categorías analíticas, como se explica en este artículo, buscaba establecer relaciones de cooperación, colaboración y reciprocidad entre actores que partían de posiciones teóricas, académicas, disciplinares y contractuales distintas. Considerando esta experiencia y las reflexiones surgidas tras ella, nos aventuramos a ir más allá de la crítica, esbozando hibridaciones metodológicas que incorporen la dimensión colectiva a la producción de conocimiento. En nuestro caso, consideramos que la co-construcción de la guía de campo fue un proceso marcado por la ética inherente a la metodología IAP, favoreciendo resultados más equitativos, o, como mínimo, ganando legitimidad en la medida que se desarrolló de forma conjunta. Queda por averiguar, y es tema que debe ser tratado en elaboraciones posteriores, el impacto de estos cambios metodológicos en el resultado de las investigaciones que estamos proponiendo.

Desde este grupo se comparte la idea de que más allá de cumplir con determinados objetivos político-epistemológicos, este sistema de trabajo ha supuesto una transformación de nuestras prácticas de investigación. En primer lugar, las categorías de la guía de campo se han seleccionado de forma reflexiva, en un proceso de visibilización consciente de algunas relaciones de poder objetivas y objetivadas dentro del proyecto ParticiPat. Sin embargo, tampoco queremos idealizar la IAP como si fuera la panacea definitiva contra las asimetrías académicas, o esencializarla como si fuera necesariamente una metodología autónoma de un contexto social donde preexisten, coexisten y prevalecen lógicas intersectadas de dominación. Más bien la IAP ha sido en nuestro caso una herramienta que ha contribuido a identificar estructuras de desigualdad internas, poniendo en primer plano a aquellas investigadoras con posiciones más inestables y voces más inaudibles.

En este artículo, hemos descrito las micropolíticas del diseño colaborativo de un proyecto, sabiendo que para minimizar las relaciones de poder en el campo académico no solamente debemos replantear las prácticas de investigación, sino también cuestionar la organización del campo intelectual y la orientación de las políticas científicas. La oportunidad de seguir experimentando con formas colaborativas de construcción de saberes, así como la posibilidad misma de democratizar los marcos metodológicos de la disciplina, se vinculan en buena medida con el compromiso que asumamos en procesos más amplios de transformación social.

\section{AGRADECIMIENTOS}

Queremos agradecer especialmente las aportaciones de todas las investigadoras presentes en la gestación del artículo: Victoria Quintero, Nieves Herrero, Guadalupe Jiménez Esquinas, David González, María Masaguer y José Muñoz. También queremos reconocer la participación en la redacción del manuscrito final de Guadalupe Jiménez Esquinas y Virtudes Téllez Delgado ${ }^{1}$. Lamentamos especialmente el caso de Virtudes Téllez

\footnotetext{
${ }^{1} N$. de la R. Las normas de la revista indican que, salvo casos excepcionales, no se publicarán artículos de un mismo autor(a) con una periodicidad inferior a tres años.
} 
que no puede aparecer como firmante por imponderables editoriales que escapan a nuestra voluntad. Deseamos señalar que la escritura del artículo ha sido un proceso complejo y doloroso donde se han manifestado los límites, riesgos y contradicciones de nuestra propuesta metodológica. Nuestra apuesta inicial por la heterarquía ha tenido efectos indeseados y desgastantes para el grupo en su conjunto y hubiera requerido de otros tiempos, espacios y disposiciones para su concreción satisfactoria. En este sentido, consideramos que la reproducción de la ortodoxia autoritaria en muchos grupos de investigación no se puede explicar únicamente como una forma de dominación política, sino como una respuesta en cierta medida adaptativa al contexto vigente de producción de conocimiento. En última instancia, la normatividad académica hegemónica ha prevalecido, generando conflictos de intereses entre las autoras del artículo con sus propias aspiraciones personales en el campo académico. La incomodidad vivida en los momentos de negociación de la autoría debería advertirnos de la tremenda necesidad de ubicarnos a nosotras mismas dentro de las estructuras disciplinares que procuramos desestabilizar. La materialización de las prácticas colaborativas requiere de enorme reflexividad y solamente es posible en el plano realmente existente de la reciprocidad, la cotidianidad y los horizontes ideológicos compartidos.

\section{BIBLIOGRAFÍA CITADA}

Ball, Stephen. 1989. La micropolítica de la escuela. Hacia una teoría de la organización escolar. Madrid: Paidós/MEC.

Berraquero Díaz, Luis, Francisco J. Maya Rodríguez y Francisco J. Escalera. 2016. "La colaboración como condición: la etnografía participativa como oportunidad para la acción". Revista de Dialectología y Tradiciones Populares 71(1): 49-57.

Bourdieu, Pierre. 2002. Interventions politiques, 1961-2000: science sociale et action politique. Marsella/Montreal: Agone/Comeau et Nadeau.

Castro, Pablo y Luís Rodríguez. 2009. "Antropología de los procesos políticos y del poder". Alteridades 19(38): 107-127.

Coca, Agustín. 2008. Los Camperos. Territorios, usos sociales y percepciones en un "espacio natural" andaluz. Sevilla: Fundación Blas Infante.

Coca, Agustín y Antonio Luis Díaz. 2005. "Protección ambiental y percepciones locales en los Parques Naturales andaluces", en José Pascual y David Florido (coords.), ¿Protegiendo los recursos? Áreas protegidas, poblaciones locales y sostenibilidad: 177-191. Sevilla: Federación de Asociaciones de Antropología del Estado Español/ Fundación El Monte/Asociación Andaluza de Antropología.

Colectivo Ioé. 2003. "Investigación acción participativa: propuesta para un ejercicio activo de la ciudadanía". Conferencia presentada en el Encuentro de la Consejería de Juventud, Córdoba 2003. Disponible en: <https://www.colectivoioe.org/uploads/89050a31b85b9e19068a9beb6db3dec136885013.pdf>. Fecha de acceso: 7 jul. 2016

Cortesão, Luiza. 2006. "Investigación acción: una invitación a prácticas científicamente transgresivas", en João Francisco de Souza (org.), Investigación Acción participativa: ¿¿qué?? Desafios a la construcción colectiva del conocimiento: 179-209. Recife: Edições Bagaço.

Cortés-Vázquez, José Antonio, Guadalupe Jiménez Esquinas y Cristina Sánchez-Carretero. 2017. "Heritage and ParticiPatory Governance. An Analysis of Political Strategies and Social Fractures in Spain". Anthropology Today 33.1: 15-18. doi: <https://doi.org/10.1111/1467-8322.12324>.

Díaz de Rada, Ángel. 2011. El taller del etnógrafo. Materiales y herramientas de investigación en etnografia. Madrid: UNED.

Escalera, Francisco J. y Agustín Coca. 2013. "Teoría y práctica de la participación”, en Javier Escalera y Agustín Coca (coords.), Movimientos sociales, participación y ciudadanía en Andalucía: 17-38. Sevilla: Aconcagua Libros. 
Fals Borda, Orlando. 1978. "Por la praxis: el problema de cómo investigar la realidad para transformarla", en Simposio Mundial de Cartagena, Crítica y politica en ciencias sociales, el debate sobre teoría y práctica, vol. 1: 209-249. Bogotá: Punta de Lanza/Universidad de Los Andes.

Fals Borda, Orlando. 1985. Conocimiento y poder popular. Bogotá: Siglo XXI/Punta de Lanza.

Freire, Paulo. 1997 [1970]. Pedagogía del oprimido. Madrid: Siglo XXI.

Greenwood, Davyd y Morten Levin. 2007. Introduction to Action Research. Social research for social change. Londres/Nueva Delhi: Thousand Oaks/Sage Publications.

Grosfoguel, Ramon. 2013. "Racismo/sexismo epistémico, universidades occidentalizadas y los cuatro genocidios/epistemicidios del largo siglo XVI". Tabula Rasa 19: 31-58.

Guattari Félix y Suely Rolnik. 2006. Micropolítica. Cartografía del deseo. Buenos Aires: Tinta limón.

Heron, John. 1996. Co-operative inquiry: research into human condition. Londres: Sage publication.

Ibáñez, Jesús. 1985. Del algoritmo al sujeto. Madrid: Siglo XXI.

Lamphere, Louise. 2004. "The Convergence of Applied, Practicing, and Public Anthropology in the 21st Century". Human Organization 63(4): 431-443.

Lassiter, Eric. 2005. The Chicago Guide to Collaborative Ethnography. Chicago: University of Chicago Press.

Low, Setha y Sally Merry. 2010. "Engaged Anthropology: Diversity and Dilemmas". Current Anthropo$\log$ 51(2): 203-226.

Lucio Villegas, Emilio Luis. 2006. "Investigación acción: una invitación a prácticas científicamente transgresivas", en João Francisco de Souza (org.), Investigación Acción participativa: ¿¿qué?? Desafíos a la construcción colectiva del conocimiento: 147-178. Recife: Edições Bagaço.

De Souza, João Francisco F. "Investigación Acción participativa: realidades y desafíos", en João Francisco de Souza (org.), Investigación Acción participativa: ¿¿qué?? Desafíos a la construcción colectiva del conocimiento: 108 146. Recife: Edições Bagaço.

Marshall, Judi. 2004. "Living sistematic thinking". Journal of Action Research 2(3): 305-325. doi: <https:/ /doi.org/10.1177/1476750304045945>.

Pérez Orozco, Amaia. 2014. Subversión feminista de la economía. Aportes para un debate sobre el conflicto capital vida. Madrid: Traficantes de Sueños.

Purcell, Trevor. 2000. "Public Anthropology: An Idea Searching for a Reality". Transforming Anthropology 9: 30-33. doi: <https://doi.org/10.1525/tran.2000.9.2.30>.

Quintero, Victoria. 2011. "El patrimonio pertenece a todos”. De la universalidad a la identidad, ¿cuál es el lugar de la participación social?", en Iñaki Arrieta Urtizberea (ed.), Legitimaciones sociales de las políticas patrimoniales y museísticas: 45-78. Bilbao: Universidad del País Vasco.

Reason, Peter. 1994. "Towards a ParticiPatory Worldview", en Peter Reason (ed.), ParticiPation in Human Inquiry: 16-39. Londres: Sage.

Red CIMAS. 2015. Metodologías participativas. Sociopraxis para la creatividad social. Madrid: Dextra Editorial.

Restrepo, Eduardo y Arturo Escobar. 2005. "Other Anthropologies and Anthropology Otherwise. Steps to a World Anthropologies Framework". Critique of Antbropology 25(2): 99-129. doi: <https:// doi.org/10.1177/0308275X05053009>.

Rodrigues Brandão, Carlos. 1986. Pesquisa participante. San Pablo: Brasiliense.

Sánchez Carretero, Cristina. 2013. "Significance and Social Value of Cultural Heritage: Analyzing the Fractures of Heritage», en Miguel Ángel Rogerio Candelera, Maurizio Larazzi y Emilio Cano (eds.), Science and Technology for the Conservation of Cultural Heritage: 387-392. Londres: Taylor \& Francis.

Sánchez Carretero, Cristina y Guadalupe Jiménez Esquinas. 2016. "Relaciones entre actores patrimoniales: gobernanza patrimonial, modelos neoliberales y procesos participativos". Revista PH 90: 190-197.

Schensul, Jean J. y Stephen L. Schensul. 1978. "Advocacy and applied anthropology", en George H. Weber y George J. McCall (eds.), Social scientists as advocates: views from the applied disciplines: 121-164. Beverly Hills: Sage Publications.

Schensul, Jean J. y Stephen L. Schensul. 1992. "Collaborative research: methods of inquiry for social Change", en Margaret Diane LeCompte, Wendy L. Millroy y Judith Preissle (eds.), Handbook of qualitative research in education: 161-200. San Diego: Academic Press. 
Shukaitis, Stevphen y David Graeber (eds.). 2007. Constituent Imagination: Militant Investigation, Collective Theorization. Oakland: Ak Press.

Torbert, William. 2001. "The practice of action inquiry", en Peter Reason y Hilary Bradbury (eds.), Handbook of Action Research. ParticiPative Inquiry and practice: 250-260. Londres/Nueva Delhi: Thousand Oaks/Sage Publications.

Vejarano, Gilberto. 1983. La investigación participativa en América Latina. Antología. Pátzcuaro: Crefal.

Weber, Max. 1978. Ensayos de sociología. Madrid: Taurus.

Fecha de recepción: 5 de julio de 2017

Fecha de aceptación: 26 de enero de 2018 